\title{
Continuous flow based catch and release protocol for the synthesis of $\alpha$-ketoesters
}

\author{
Alessandro Palmieri ${ }^{1}$, Steven V. Ley ${ }^{*}, 1$, Anastasios Polyzos ${ }^{1,2}$, \\ Mark Ladlow ${ }^{3}$ and lan R. Baxendale ${ }^{1}$
}

\section{Full Research Paper}

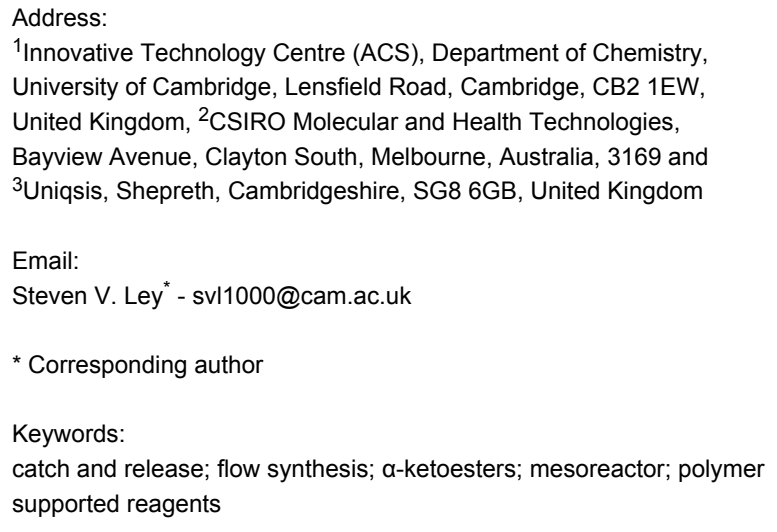

\section{Open Access}

Beilstein Journal of Organic Chemistry 2009, 5, No. 23 doi:10.3762/bjoc.5.23

Received: 05 March 2009

Accepted: 14 May 2009

Published: 20 May 2009

Guest Editor: A. Kirschning

(c) 2009 Palmieri et al; licensee Beilstein-Institut. License and terms: see end of document.

\begin{abstract}
Using a combination of commercially available mesofluidic flow equipment and tubes packed with immobilised reagents and scavengers, a new synthesis of $\alpha$-ketoesters is reported.
\end{abstract}

\section{Introduction}

Organic synthesis is changing rapidly owing to the discovery of processes that challenge current dogma and lead to the invention of new chemical reactions [1,2]. Likewise, new synthesis tools are impacting on the way we assemble molecules. Of these, flow chemistry technologies are becoming especially important [3-14]. For many years, our group [15-22] has been focussed on using immobilised systems [23-29] to more effectively and cleanly bring about chemical transformations, especially in multistep mode [17,30-37]. Given the success of these concepts, it is not surprising that we would want to adapt these principles to various flow-chemical synthesis platforms to effectuate automated multistep chemical syntheses [38-54].
In this work we report the use of the Uniqsis FlowSyn ${ }^{\mathrm{TM}}$ continuous flow reactor [55] (Figure 1) to effect a flow-based preparation of $\alpha$-ketoesters. The key feature of this process is the application of a catch and release protocol [56-72], under the flow reaction conditions. Our choice of $\alpha$-ketoesters as products of the process was governed by their use as starting materials for various synthesis programmes [73-81] and as important products in their own right [82-88]. Common methods for the preparation of $\alpha$-ketoesters include the modified Dakin-West reaction [89] and the addition of a Grignard reagent to oxalates or oxalyl chlorides [90-92] together with a few alternative syntheses [93-99]. These procedures often suffer 

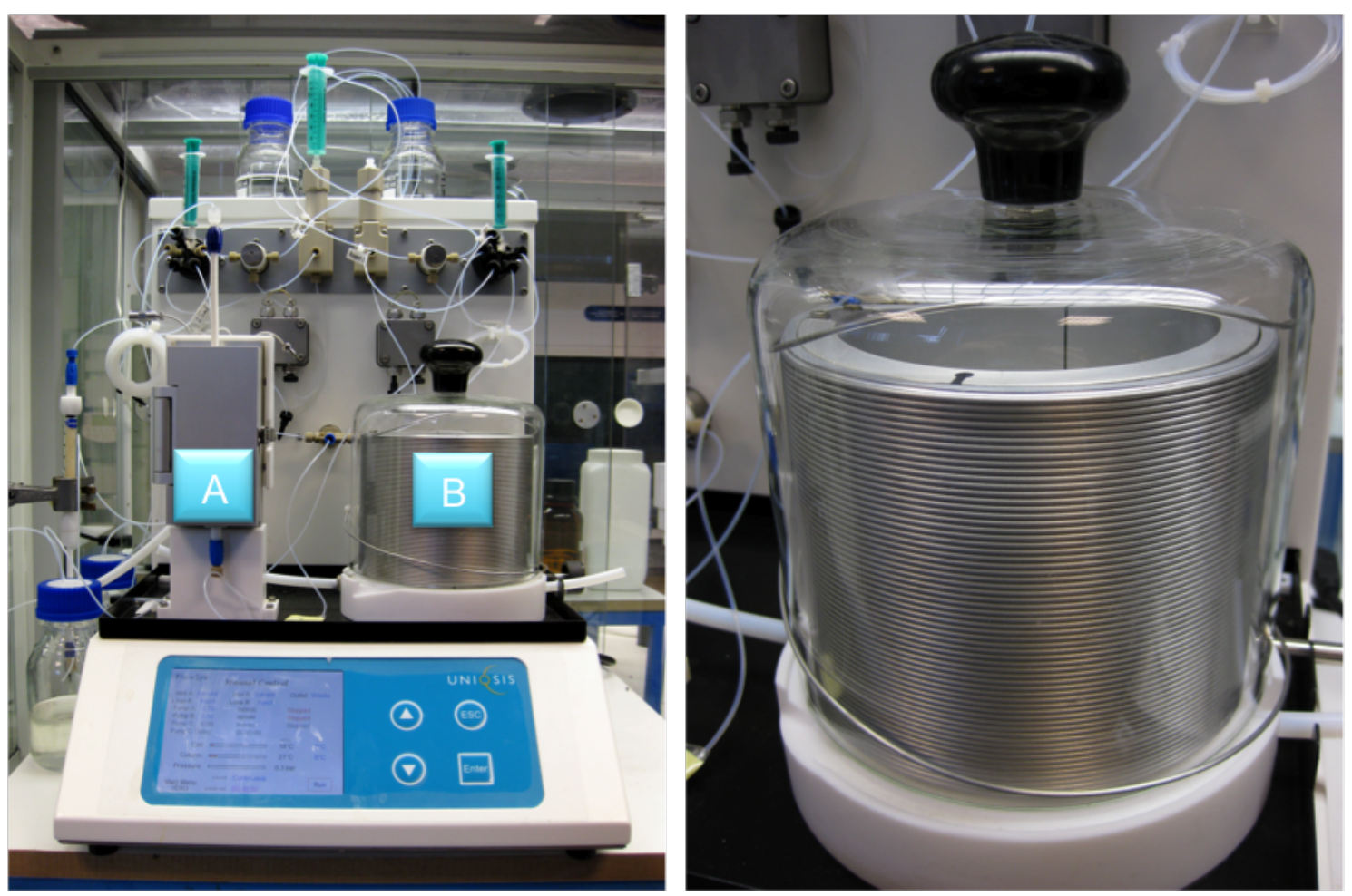

Figure 1: The Uniqsis FlowSyn ${ }^{\mathrm{TM}}$ continuous flow reactor comprising of a column holder and heating unit $(\mathrm{A})$ and the reactor coil $(\mathrm{B})$. $\mathrm{A}$ detailed image of the reactor coil is shown on the right.

from drastic conditions, restricted selectivity and poor yields. Our flow-based approach delivers a new and general method for the preparation of $\alpha$-ketoesters, which proceeds under mild conditions, with good functional group tolerability and generates products in high purity.

\section{Results and Discussion}

The experimental set up for these transformations involves the use of the Uniqsis FlowSyn ${ }^{\mathrm{TM}}$ device [55]. The fully integrated instrument employs a dual channel flow system, with each channel independently driven by a variable high-pressure piston pump. The starting materials and reagents are dispensed from sample loops $(0.5-10 \mathrm{~mL})$ and are united in a T-mixing piece and then passed into either a coil or column reactor (Figure 1). The column reactor utilises adjustable glass columns with variable internal diameter $(1-1.5 \mathrm{~cm})$ and range in volume from 6-83 mL (unpacked). The coil reactors are made from a selection of materials including PTFE, PEEK, stainless steel or Hastelloy ${ }^{\circledR}$ and accommodate volumes from $2-20 \mathrm{~mL}$. The column reactor (Figure 1, A) can be heated up to $150^{\circ} \mathrm{C}$ and the coil heater (Figure 1, B) up to $260^{\circ} \mathrm{C}$, over a range of flow rates between $0.01-20 \mathrm{~mL} / \mathrm{min}$, and can be configured for multistep or parallel operation. Exiting products can be collected as aliquots using an automated fraction collector for reaction optimisation or as a bulk sample for scale-up. In addition, product purification can be achieved as part of the overall flow process by in-line solid phase extraction (SPE) or alternatively by diverting the flow stream into an attached HPLC system [100].

A series of preliminary experiments was carried out on the flow equipment to profile the reaction in terms of optimum reaction temperature, concentration, residence time, solvent and stoichiometry. Following rapid screening of conditions, we fixed upon a set of reaction parameters for efficient synthesis of $\alpha$-ketoesters (Scheme 1). The overall reaction process proceeds in the flow apparatus via nitroolefinic esters $\mathbf{1}$ as substrates which are captured onto a benzylamine polymer 2 (QuadraPure $^{\text {TM }} \mathrm{QP}-\mathrm{BZA}$ polymer, loading $5.5 \mathrm{mmol} / \mathrm{g}, 4$ equiv) to give 3 to effect product clean-up. In this way the immobilised species 3 can be washed and any solution phase impurities (resulting from the formation of the nitroolefinic ester - see later) are directed to waste (step 1). Next the column is treated with a flow stream of tetramethylguanidine (TMG; step 2) to cause elimination of nitrous acid and produce the corresponding enamino acid esters, which remain attached to the polymer support. Finally, after flow-washing (step 3), the solid supported species is hydrolysed, liberating $\alpha$-ketoester product 4 by flowing aqueous acetic acid (step 4) through the in-line column. The overall route constitutes a new flow chemistry 
<smiles>[R]/C(=C\C(=O)OC)[N+](=O)[O-]</smiles>

$1 a-j$

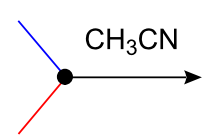

Step 1. rt, $0.2 \mathrm{~mL} / \mathrm{min}$

Step 2. $65^{\circ} \mathrm{C}$, TMG, $0.1 \mathrm{~mL} / \mathrm{min}$

Step 3. rt, $0.2 \mathrm{~mL} / \mathrm{min}$

Step 4. rt, $\mathrm{H}_{2} \mathrm{O} / \mathrm{AcOH}, 0.3 \mathrm{~mL} / \mathrm{min}$
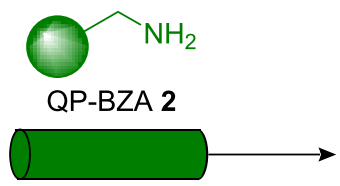

$\mathrm{rt}, 0.2 \mathrm{~mL} / \mathrm{min}$ (1)<smiles>[R]CC(=O)C(=O)OCC</smiles>

4a-j

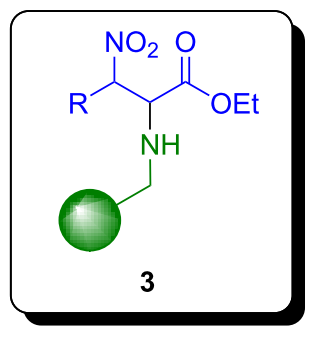

Scheme 1: General procedure for the flow synthesis of $\alpha$-ketoester products $\mathbf{4 a - j}$.

example of the catch-react-and-release concept that we have used successfully in other synthesis programmes [101-103].

The nitroolefinic esters 1 were originally formed in a separate batch reaction from a Henry coupling of appropriate nitro compounds with ethyl glyoxalate over Amberlyst ${ }^{\mathrm{TM}} 21$ (A21) resin to give the corresponding nitroalkanol 5 [104]. This was followed by treatment of $\mathbf{5}$ with methanesulfonyl chloride $(\mathrm{MsCl})$ or trifluoroacetic anhydride (TFAA) to promote the base-catalysed dehydration, affording the nitroolefinic esters 1 (Scheme 2) [105]. As we have deliberately constructed this sequence for implementation in a continuous flow process, the intermediate nitroalkanols $\mathbf{5}$ were not isolated and the nitroolefinic esters were used without further purification. The average yield for the nitroolefins $\mathbf{1 a}-\mathbf{j}$ prepared as described in Scheme 2 was approximately $60 \%$ by LCMS. Impurities were readily removed following immobilisation of nitroolefinic esters 1 on the QP-BZA resin.

In addition, the flow synthesis of two representative compounds was undertaken to allow for the complete generation of $\alpha$-ketoester products in flow from the starting nitroalkanes (Scheme 3). As shown in Table 1, we demonstrate that the synthesis of the nitroolefinic esters was achieved under flow conditions in a clean and effective fashion. Moreover, this synthesis demonstrates the first reported example of Henry reaction conducted in flow and we intend to elaborate on this important transformation in future studies.<smiles>[R]COC(=O)/C=C(/[R])NOC(C)(C)Cl</smiles>

Scheme 2: General procedure for the batch synthesis of nitroolefinic esters $1 \mathbf{a}-\mathbf{j}$.

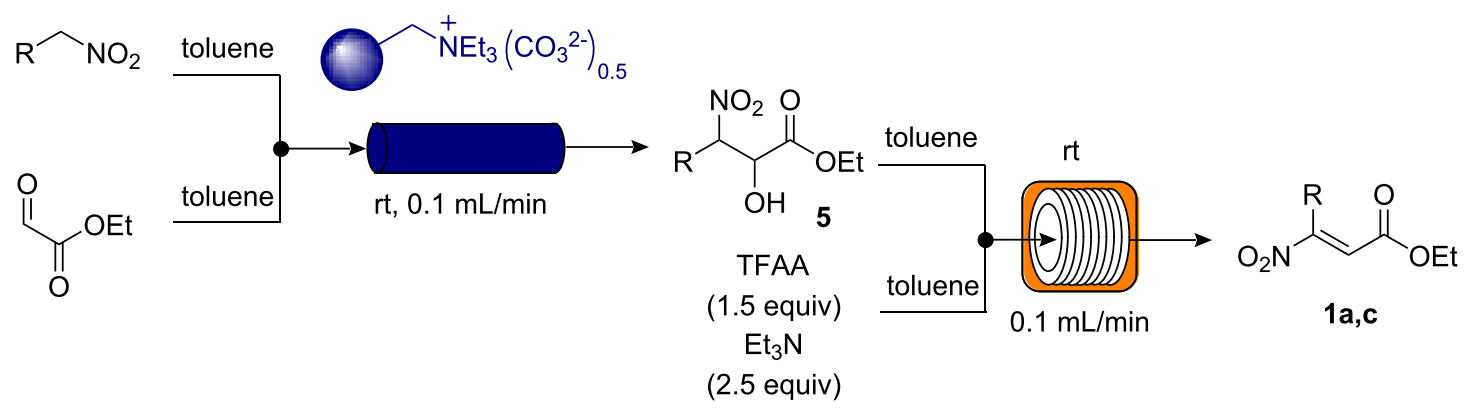

Scheme 3: General procedure for the flow synthesis of nitroolefinic esters 1a,c. 
Table 1: Nitroolefinic esters 1a,c prepared under flow conditions (as described in Scheme 3).

Entry

alsolated yields are shown.

Figure 2 illustrates the examples and yields of $\alpha$-ketoester products afforded by this new approach. While the list is not extensive, we have established that the process is tolerant of both aliphatic and aromatic substituted nitro-derivatives in the first step, and accommodates ester, acetate, acetal, nitrile and olefinic functionality in the final product. The process was reliable over several runs and consistently afforded very clean material ( $\geq 97 \%$ by NMR). The yields while only moderate for the overall process still equate to an average step conversion of $68-78 \%$ per chemical iteration, given that the sequence is a multistep process (see Supporting Information for full experimental data).

\section{Conclusion}

In conclusion, we have demonstrated the versatility of the Uniqsis FlowSyn ${ }^{\mathrm{TM}}$ unit to achieve multi-step organic synthesis under continuous flow-chemistry conditions. This was accomplished by adapting the device to incorporate immobilised reagents packed in flow tubes, enabling clean transformations without recourse to conventional product work-up or purification. The overall process delivers synthetically useful $\alpha$-ketoester products in high purity from various nitroalkane inputs and paves the way for more extended reaction sequences.

\section{Supporting Information}

\section{Supporting Information File 1}

Supporting Information - Continuous flow based catch and release protocol for the synthesis of $\alpha$-ketoesters [http://www.beilstein-journals.org/bjoc/content/ supplementary/1860-5397-5-23-S1.doc]

\section{Acknowledgments}

We gratefully acknowledge financial support from the EPRSC (to I. R. Baxendale), the BP endowment (to S. V. Ley), University of Camerino and MIUR-Italy (to A. Palmieri) and CSIRO Capability Development Fund (CDF) (to A. Polyzos).

\section{References}

1. Ley, S. V.; Baxendale, I. R. Nat. Rev. Drug Discovery 2002, 1, 573-586. doi:10.1038/nrd871

2. Baxendale, I. R.; Hayward, J. J.; Ley, S. V.; Tranmer, G. K. ChemMedChem 2007, 2, 768-788. doi:10.1002/cmdc.200700008

3. Baxendale, I. R.; Pitts, M. R. Chim. Oggi 2006, 24 (3), 41-45.

4. Baxendale, I. R.; Ley, S. V. Heterogeneous Reactions. In New Avenues to Efficient Chemical Synthesis, Emerging Technologies; Seeberger, P. H.; Blume, T., Eds.; Springer-Verlag: Berlin, Heidelberg, 2007; pp 151-185.

5. Baxendale, I. R.; Hayward, J. J.; Ley, S. V. Comb. Chem. High Throughput Screening 2007, 10, 802-836. doi:10.2174/138620707783220374

6. Baxendale, I. R.; Hayward, J. J.; Lanners, S.; Ley, S. V.; Smith, C. D. Heterogeneous Reactions. In Microreactors in Organic Synthesis and Catalysis; Wirth, T., Ed.; Wiley-VCH: Weinheim, 2008; pp 84-122. Chapter 4.2 .

7. Jas, G.; Kirschning, A. Chem.-Eur. J. 2003, 9, 5708-5723. doi:10.1002/chem.200305212

8. Hodge, P. Curr. Opin. Chem. Biol. 2003, 7, 362-373. doi:10.1016/S1367-5931(03)00052-8

9. Jähnisch, K.; Hessel, V.; Löwe, H.; Baerns, M. Angew. Chem. 2004, 116, 410-451. doi:10.1002/ange.200300577 Angew. Chem., Int. Ed. 2004, 43, 406-446. doi:10.1002/anie.200300577.\footnotetext{
<smiles>CCCC(=O)C(=O)OCC</smiles>

$4 a, 36 \%$<smiles>CCCC(=O)C(=O)OCC</smiles>

4b, $45 \%$<smiles>CCOC(=O)C(=O)CCc1ccccc1</smiles>

4c, $44 \%$<smiles>CCOC(=O)CCCC(=O)OC</smiles>

$4 d, 31 \%$<smiles>CCOC(=O)C(=O)CCOC(C)=O</smiles>

$4 e, 44 \%$<smiles>CCOC(=O)C(=O)CCC1OCCCO1</smiles>

4f, $42 \%$<smiles>CCCCC(=O)C(=O)OCC</smiles>

4g, $43 \%$<smiles>C=CCCC(=O)C(=O)OCC</smiles>

4h, $45 \%$<smiles>CCOC(=O)C(=O)CCC(C)C</smiles>

4i, $41 \%$<smiles>CCOC(=O)C(=O)CC#N</smiles>

$4 j, 48 \%$

Figure 2: a-Ketoesters prepared and isolated yields.
} 
10. Kirschning, A.; Solodenko, W.; Mennecke, K. Chem.-Eur. J. 2006, 12, 5972-5990. doi:10.1002/chem.200600236

11. Ahmed-Omer, B.; Brandt, J. C.; Wirth, T. Org. Biomol. Chem. 2007, 5, 733-740. doi:10.1039/b615072a

12. Mason, B. P.; Price, K. E.; Steinbacher, J. L.; Bogdan, A. R.; McQuade, D. T. Chem. Rev. 2007, 107, 2300-2318. doi:10.1021/cr050944c

13. Glasnov, V. T. N.; Kappe, C. O. Macromol. Rapid Commun. 2007, 28, 395-410. doi:10.1002/marc.200600665

14. Benito-López, F.; Egberink, R. J. M.; Reinhoudt, D. N.; Verboom, W. Tetrahedron 2008, 64, 10023-10040. doi:10.1016/j.tet.2008.07.108

15. Ley, S. V.; Baxendale, I. R.; Bream, R. N.; Jackson, P. S.; Leach, A. G.; Longbottom, D. A.; Nesi, M.; Scott, J. S.; Storer, R. I.; Taylor, S. J. J. Chem. Soc., Perkin Trans. 1 2000, 3815-4195. doi:10.1039/b006588i

16. Baxendale, I. R.; Lee, A.-L.; Ley, S. V. J. Chem. Soc., Perkin Trans. 1 2002, 1850-1857. doi:10.1039/b203388g

17. Baxendale, I. R.; Ernst, M.; Krahnert, W.-R.; Ley, S. V. Synlett 2002, 1641-1644. doi:10.1055/s-2002-34249

18. Ley, S. V.; Baxendale, I. R. Chem. Rec. 2002, 2, 377-388. doi:10.1002/tcr.10033

19. Baxendale, I. R.; Ley, S. V.; Nesi, M.; Piutti, C. Tetrahedron 2002, 58, 6285-6304. doi:10.1016/S0040-4020(02)00628-2

20. Storer, R. I.; Takemoto, T.; Jackson, P. S.; Ley, S. V. Angew. Chem. 2003, 115, 2625-2629. doi:10.1002/ange.200351413 Angew. Chem., Int. Ed. 2003, 42, 2521-2525. doi:10.1002/anie.200351413

21. Storer, R. I.; Takemoto, T.; Jackson, P. S.; Brown, D. S.; Baxendale, I. R.; Ley, S. V. Chem.-Eur. J. 2004, 10, 2529-2547. doi:10.1002/chem.200305669

22. Baxendale, I. R.; Ley, S. V. Ind. Eng. Chem. Res. 2005, 44, 8588-8592. doi:10.1021/ie048822i

23. Thompson, L. A. Curr. Opin. Chem. Biol. 2000, 4, 324-337. doi:10.1016/S1367-5931(00)00096-X

24. Kobayashi, S. Curr. Opin. Chem. Biol. 2000, 4, 338-345. doi:10.1016/S1367-5931(00)00097-1

25. Kirschning, A.; Monenschein, H.; Wittenberg, R. Chem.-Eur. J. 2000, 6, 4445-4450.

doi:10.1002/1521-3765(20001215)6:24<4445::AID-CHEM4445>3.0.C O;2-W

26. Kirschning, A.; Monenschein, H.; Wittenberg, R. Angew. Chem. 2001, 113, 670-701.

doi:10.1002/1521-3757(20010216)113:4<670::AID-ANGE6700>3.0.C $0 ; 2-\mathrm{G}$

Angew. Chem., Int. Ed. 2001, 40, 650-679.

doi:10.1002/1521-3773(20010216)40:4<650::AID-ANIE6500>3.0.CO; 2-C.

27. Sherrington, D. C. J. Polym. Sci., Part A: Polym. Chem. 2001, 39, 2364-2377. doi:10.1002/pola.1213

28. Hodge, P. Ind. Eng. Chem. Res. 2005, 44, 8542-8553. doi:10.1021/ie040285e

29. Solinas, A.; Taddei, M. Synthesis 2007, 2409-2453. doi:10.1055/s-2007-983806

30. Baxendale, I. R.; Ley, S. V. Bioorg. Med. Chem. Lett. 2000, 10, 1983-1986. doi:10.1016/S0960-894X(00)00383-8

31. Ley, S. V.; Baxendale, I. R.; Brusotti, G.; Caldarelli, M.; Massi, A.; Nesi, M. Farmaco 2002, 57, 321-330. doi:10.1016/S0014-827X(02)01210-7

32. Baxendale, I. R.; Brusotti, G.; Matsuoka, M.; Ley, S. V. J. Chem. Soc., Perkin Trans. 1 2002, 143-154. doi:10.1039/b109482n
33. Baxendale, I. R.; Lee, A.-L.; Ley, S. V. Synlett 2002, 516-518. doi:10.1055/s-2002-20483

34. Baxendale, I. R.; Ley, S. V.; Lumeras, W.; Nesi, M. Comb. Chem. High Throughput Screening 2002, 5, 197-199.

35. Baxendale, I. R.; Ley, S. V.; Sneddon, H. F. Synlett 2002, 775-777. doi:10.1055/s-2002-25333

36. Baxendale, I. R.; Storer, R. I.; Ley, S. V. Supported Reagents and Scavengers in Multi-Step Organic Synthesis. In Polymeric Materials in Organic Synthesis and Catalysis; Buchmeiser, M. R., Ed.; Wiley-VCH: Weinheim, 2003; pp 53-136. doi:10.1002/3527601856.ch2

37. Baxendale, I. R.; Ley, S. V. Curr. Org. Chem. 2005, 9, 1521-1534. doi:10.2174/138527205774370513

38. Baxendale, I. R.; Deeley, J.; Griffiths-Jones, C. M.; Ley, S. V.; Saaby, S.; Tranmer, G. K. Chem. Commun. 2006, 2566-2568. doi:10.1039/b600382f

39. Baumann, M.; Baxendale, I. R.; Ley, S. V.; Smith, C. D.; Tranmer, G. K. Org. Lett. 2006, 8, 5231-5234. doi:10.1021/ol061975c

40. Smith, C. J.; Iglesias-Sigüenza, F. J.; Baxendale, I. R.; Ley, S. V. Org. Biomol. Chem. 2007, 5, 2758-2761. doi:10.1039/b709043a

41. Smith, C. D.; Baxendale, I. R.; Lanners, S.; Hayward, J. J.; Smith, S. C.; Ley, S. V. Org. Biomol. Chem. 2007, 5, 1559-1561. doi:10.1039/b702995k

42. Hornung, C. H.; Mackley, M. R.; Baxendale, I. R.; Ley, S. V. Org. Process Res. Dev. 2007, 11, 399-405. doi:10.1021/op700015f

43. Baumann, M.; Baxendale, I. R.; Ley, S. V. Synlett 2008, 2111-2114 doi:10.1055/s-2008-1078026

44. Baumann, M.; Baxendale, I. R.; Ley, S. V.; Nikbin, N.; Smith, C. D. Org. Biomol. Chem. 2008, 6, 1587-1593. doi:10.1039/b801634h

45. Baumann, M.; Baxendale, I. R.; Ley, S. V.; Nikbin, N.; Smith, C. D.; Tierney, J. P. Org. Biomol. Chem. 2008, 6, 1577-1586. doi:10.1039/b801631n

46. Baxendale, I. R.; Ley, S. V.; Smith, C. D.; Tamborini, L.; Voica, A.-F. J. Comb. Chem. 2008, 10, 851-857. doi:10.1021/cc800070a

47. Jas, G.; Kirschning, A. Chem.-Eur. J. 2003, 9, 5708-5723. doi:10.1002/chem.200305212

48. Bernstein, D.; France, S.; Wolfer, J.; Lectka, T. Tetrahedron: Asymmetry 2005, 16, 3481-3483. doi:10.1016/j.tetasy.2005.09.014

49. Bonfils, F.; Cazaux, I.; Hodge, P.; Caze, C. Org. Biomol. Chem. 2006, 4, 493-497. doi:10.1039/b515241k

50. Wiles, C.; Watts, P.; Haswell, S. J. Tetrahedron Lett. 2006, 47, 5261-5264. doi:10.1016/j.tetlet.2006.05.157

51. Dräger, G.; Kiss, C.; Kunz, U.; Kirschning, A. Org. Biomol. Chem. 2007, 5, 3657-3664. doi:10.1039/b712804e

52. Burguete, M. I.; Cornejo, A.; García-Verdugo, E.; Gil, M. J.; Luis, S. V.; Mayoral, J. A.; Martínez-Merino, V.; Sokolova, M. J. Org. Chem. 2007, 72, 4344-4350. doi:10.1021/jo070119r

53. Solodenko, W.; Jas, G.; Kunz, U.; Kirschning, A. Synthesis 2007, 583-589. doi:10.1055/s-2007-965877

54. Odedra, A.; Geyer, K.; Gustafsson, T.; Gilmour, R.; Seeberger, P. H. Chem. Commun. 2008, 3025-3027. doi:10.1039/b803715a

55. Uniqsis web site. http://www.uniqsis.com (accessed Apr 6, 2009).

56. Cohen, B. J.; Kraus, M. A.; Patchornik, A. J. Am. Chem. Soc. 1977, 99, 4165-4167. doi:10.1021/ja00454a050

57. Cohen, B. J.; Kraus, M. A.; Patchornik, A. J. Am. Chem. Soc. 1981, 103, 7620-7629. doi:10.1021/ja00415a034

58. Patchornik, A. In Proc. IUPAC, I. U. P. A. C., Macromol. Symp., 28th, 1982, University of Massachusetts, Amherst, July 12-16, 1982; IUPAC: Oxford, 1982; p 85. 
59. Brown, S. D.; Armstrong, R. W. J. Am. Chem. Soc. 1996, 118, 6331-6332. doi:10.1021/ja961203j

60. Hu, Y.; Baudart, S.; Porco, J. A., Jr. J. Org. Chem. 1999, 64, 1049-1051. doi:10.1021/jo981874v

61. Studer, A.; Hadida, S.; Ferritto, R.; Kim, S.-Y.; Jeger, P.; Wipf, P.; Curran, D. P. Science 1997, 275, 823-826.

doi:10.1126/science.275.5301.823

62. Flynn, D. L.; Crich, J. Z.; Devraj, R. V.; Hockerman, S. L.; Parlow, J. J.; South, M. S.; Woodard, S. J. Am. Chem. Soc. 1997, 119, 4874-4881. doi:10.1021/ja963462e

63. Curran, D. P. Angew. Chem. 1998, 110, 1230-1255. doi:10.1002/(SICI)1521-3757(19980504)110:9<1230::AID-ANGE1230 $>3.0 . \mathrm{CO} ; 2-\mathrm{Y}$

Angew. Chem., Int. Ed. 1998, 37, 1174-1196.

doi:10.1002/(SICI)1521-3773(19980518)37:9<1174::AID-ANIE1174>3 .0.CO;2-P.

64. Bosanac, T.; Yang, J.; Wilcox, C. S. Angew. Chem. 2001, 113, 1927-1931. doi:10.1002/1521-3757(20010518)113:10<1927::AID-ANGE1927>3.0 CO;2-\#

Angew. Chem., Int. Ed. 2001, 40, 1875-1879. doi:10.1002/1521-3773(20010518)40:10<1875::AID-ANIE1875>3.0.C O;2-5.

65. Ley, S. V.; Massi, A.; Rodríguez, F.; Horwell, D. C.; Lewthwaite, R. A.; Pritchard, M. C.; Reid, A. M. Angew. Chem. 2001, 113, 1088-1090. doi:10.1002/1521-3757(20010316)113:6<1088::AID-ANGE10880>3.0. CO;2-\# Angew. Chem., Int. Ed. 2001, 40, 1053-1055. doi:10.1002/1521-3773(20010316)40:6<1053::AID-ANIE10530>3.0.C O;2-D.

66. Galante, A.; Lhoste, P.; Sinou, D. Tetrahedron Lett. 2001, 42, 5425-5427. doi:10.1016/S0040-4039(01)01055-3

67. Yoshida, J.-i.; Itami, K. Chem. Rev. 2002, 102, 3693-3716. doi:10.1021/cr0103524

68. Dobbs, A. P.; McGregor-Johnson, C. Tetrahedron Lett. 2002, 43, 2807-2810. doi:10.1016/S0040-4039(02)00322-2

69. Lan, P.; Porco, J. A., Jr.; South, M. S.; Parlow, J. J. J. Comb. Chem. 2003, 5, 660-669. doi:10.1021/cc030028h

70. Siu, J.; Baxendale, I. R.; Lewthwaite, R. A.; Ley, S. V. Org. Biomol. Chem. 2005, 3, 3140-3160. doi:10.1039/b503778f

71. Curran, D. P.; Wang, X.; Zhang, Q. J. Org. Chem. 2005, 70, 3716-3719. doi:10.1021/jo050116j

72. Mothana, S.; Chahal, N.; Vanneste, S.; Hall, D. G. J. Comb. Chem. 2007, 9, 193-196. doi:10.1021/cc060149s

73. Audrain, H.; Thorhauge, J.; Hazell, R. G.; Jørgensen, K. A. J. Org. Chem. 2000, 65, 4487-4497. doi:10.1021/jo9918596

74. Peng, Z.-H.; Woerpel, K. A. Org. Lett. 2002, 4, 2945-2948. doi:10.1021/ol026343e

75. Yu, S.; Saenz, J.; Srirangam, J. K. J. Org. Chem. 2002, 67, 1699-1702. doi:10.1021/jo016131f

76. Griesbeck, A. G.; Bondock, S.; Lex, J. Org. Biomol. Chem. 2004, 2 , 1113-1115. doi:10.1039/b401990c

77. Sun, Y.; Wan, X.; Wang, J.; Meng, Q.; Zhang, H.; Jiang, L.; Zhang, Z. Org. Lett. 2005, 7, 5425-5427. doi:10.1021/ol052212c

78. Zhang, W.; Shi, M. Chem. Commun. 2006, 1218-1220. doi:10.1039/b516467b

79. Howard, B. E.; Woerpel, K. A. Org. Lett. 2007, 9, 4651-4653. doi:10.1021/ol702148x

80. Kratzer, R.; Nidetzky, B. Chem. Commun. 2007, 1047-1049. doi:10.1039/b616475g
81. Ntaganda, R.; Milovic, T.; Tiburcio, J.; Thadani, A. N. Chem. Commun. 2008, 4052-4054. doi:10.1039/b808302a

82. Peet, N. P.; Burkhart, J. P.; Angelastro, M. R.; Giroux, E. L.; Mehdi, S.; Bey, P.; Kolb, M.; Neises, B.; Schirlin, D. J. Med. Chem. 1990, 33, 394-407. doi:10.1021/jm00163a063

83. Patel, D. V.; Rielly-Gauvin, K.; Ryono, D. E.; Free, C. A.; Smith, S. A.; Petrillo, E. W., Jr. J. Med. Chem. 1993, 36, 2431-2447. doi:10.1021/jm00069a001

84. Li, Z.; Patil, G. S.; Golubski, Z. E.; Hori, H.; Tehrani, K.; Foreman, J. E.; Eveleth, D. D.; Bartus, R. T.; Powers, J. C. J. Med. Chem. 1993, 36, 3472-3480. doi:10.1021/jm00074a031

85. Koutek, B.; Prestwich, G. D.; Howlett, A. C.; Chin, S. A.; Salehani, D.; Akhavan, N.; Deutsch, D. G. J. Biol. Chem. 1994, 269, 22937-22940.

86. Conde-Frieboes, K.; Reynolds, L. J.; Lio, Y.-C.; Hale, M. R.; Wasserman, H. H.; Dennis, E. A. J. Am. Chem. Soc. 1996, 118, 5519-5525. doi:10.1021/ja953553w

87. Otto, H.-H.; Schirmeister, T. Chem. Rev. 1997, 97, 133-172. doi:10.1021/cr950025u

88. Choe, Y.; Brinen, L. S.; Price, M. S.; Engel, J. C.; Lange, M.; Grisostomi, C.; Weston, S. G.; Pallai, P. V.; Cheng, H.; Hardy, L. W.; Hartsough, D. S.; McMakin, M.; Tilton, R. F.; Baldino, C. M.; Craik, C. S. Bioorg. Med. Chem. 2005, 13, 2141-2156. doi:10.1016/j.bmc.2004.12.053

89. Buchanan, G. L. Chem. Soc. Rev. 1988, 17, 91-109. doi:10.1039/cs9881700091

90. Nimitz, J. S.; Mosher, H. S. J. Org. Chem. 1981, 46, 211-213. doi:10.1021/jo00314a057

91. Creary, X.; Mehrsheikh-Mohammadi, M. E. J. Org. Chem. 1986, 51, 2664-2668. doi:10.1021/jo00364a009

92. Babudri, F.; Fiandanese, V.; Marchese, G.; Punzi, A. Tetrahedron 1996, 52, 13513-13520. doi:10.1016/0040-4020(96)00805-8

93. Wasserman, H. H.; Ives, J. L. J. Org. Chem. 1985, 50, 3573-3580. doi:10.1021/jo00219a025

94. Bulman Page, P. C.; Rosenthal, S. Tetrahedron Lett. 1986, 27, 1947-1950. doi:10.1016/S0040-4039(00)84419-6

95. Sakakura, T.; Yamashita, H.; Kobayashi, T.-a.; Hayashi, T.; Tanaka, M. J. Org. Chem. 1987, 52, 5733-5740. doi:10.1021/jo00235a017

96. Wong, M.-K.; Yu, C.-W.; Yuen, W.-H.; Yang, D. J. Org. Chem. 2001, 66, 3606-3609. doi:10.1021/jo0015974

97. Li, L.-S.; Wu, Y.-L. Tetrahedron Lett. 2002, 43, 2427-2430. doi:10.1016/S0040-4039(02)00290-3

98. Ma, M.; Li, C.; Peng, L.; Xie, F.; Zhang, X.; Wang, J. Tetrahedron Lett. 2005, 46, 3927-3929. doi:10.1016/j.tetlet.2005.03.199

99. Shimizu, H.; Murakami, M. Chem. Commun. 2007, 2855-2857. doi:10.1039/b704105e

100. Baxendale, I. R.; Griffiths-Jones, C. M.; Ley, S. V.; Tranmer, G. K. Synlett 2006, 427-430. doi:10.1055/s-2006-926244

101.Baxendale, I. R.; Ley, S. V.; Smith, C. D.; Tranmer, G. K. Chem. Commun. 2006, 4835-4837. doi:10.1039/b612197g

102.Smith, C. D.; Baxendale, I. R.; Tranmer, G. K.; Baumann, M.; Smith, S. C.; Lewthwaite, R. A.; Ley, S. V. Org. Biomol. Chem. 2007, 5, 1562-1568. doi:10.1039/b703033a

103. Griffiths-Jones, C. M.; Hopkin, M. D.; Jönsson, D.; Ley, S. V.; Tapolczay, D. J.; Vickerstaffe, E.; Ladlow, M. J. Comb. Chem. 2007, 9, 422-430. doi:10.1021/cc060152b

104.Ballini, R.; Bosica, G.; Forconi, P. Tetrahedron 1996, 52, 1677-1684. doi:10.1016/0040-4020(95)00996-5

105. Ballini, R.; Fiorini, D.; Palmieri, A. Tetrahedron Lett. 2004, 45, 7027-7029. doi:10.1016/j.tetlet.2004.07.141 


\section{License and Terms}

This is an Open Access article under the terms of the Creative Commons Attribution License

(http://creativecommons.org/licenses/by/2.0), which permits unrestricted use, distribution, and reproduction in any medium, provided the original work is properly cited.

The license is subject to the Beilstein Journal of Organic Chemistry terms and conditions:

(http://www.beilstein-journals.org/bjoc)

The definitive version of this article is the electronic one which can be found at:

doi:10.3762/bjoc.5.23 